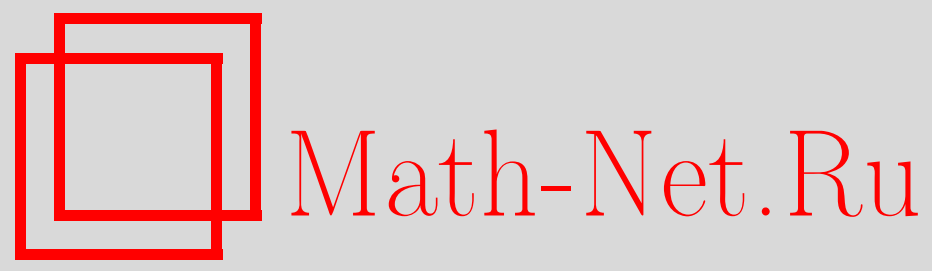

М. В. Ларин, Транзитивные полиномиальные преобразования колец вычетов, Дискрет. матем., 2002, том 14, выпуск $2,20-32$

DOI: https://doi.org/10.4213/dm238

Использование Общероссийского математического портала Math-Net.Ru подразумевает, что вы прочитали и согласны с пользовательским соглашением http: //www . mathnet.ru/rus/agreement

Параметры загрузки:

IP: 3.91 .87 .62

26 апреля 2023 г., $17: 26: 52$

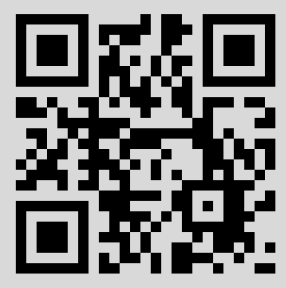


Удк 512.62

\title{
Транзитивные полиномиальные преобразования колец вычетов
}

\author{
() 2002 г. М. В. Ларин
}

\begin{abstract}
Дается полное описание полиномов $f(x)$ с целочисленными коэффициентами, для которых рекуррентная последовательность $u_{i+1} \equiv f\left(u_{i}\right)(\bmod m)$ имеет период, равный $m$.
\end{abstract}

\section{1. Введение}

1.1. Одна из центральных проблем, связанных с генераторами псевдослучайных последовательностей вида

$$
u_{i+1} \equiv f\left(u_{i}\right) \quad(\bmod m)
$$

где $u_{i} \in \mathbf{Z}, f(x) \in \mathbf{Z}[x]$, состоит в описании всех полиномов $f(x)$, для которых период последовательности (1) максимален, то есть равен $m$.

В настоящей статье будут непосредственно использованы следующие результаты, которые можно найти, например, в [1].

Предложение 1. Пусть $f(x)=a x+c$, тогда последовательность (1) имеет период, равный $m$, тогда и только тогда, когда выполнены следующие условия:

$-(c, m)=1$

- $a \equiv 1(\bmod p)$ для любого простого делителя $p$ числа $m$;

- $a \equiv 1(\bmod 4)$, если т кратно 4.

Предложение 2. Пусть $f(x)=d x^{2}+a x+c$, тогда последовательность (1) имеет период, равный m, тогда и только тогда, когда выполнены следующие условия:

$-(c, m)=1$

$-d \equiv 0(\bmod p)$ и $a \equiv 1(\bmod p)$ для любого простого нечетного делителя $p$ числа $m$;

- если т кратно 4, то $d \equiv 0(\bmod 2) u d \equiv a-1(\bmod 4)$;

- если т кратно $2, \operatorname{mo} d \equiv a-1(\bmod 2)$;

- если т кратно 9, то либо $d \equiv 0(\bmod 9)$, либо $c d \equiv 6(\bmod 9)$. 
Предложение 3. Пусть $m=2^{e}$, тогда последовательность (1) имеет период, равный $m=2^{e}$, если для полинома $f(x) \in \mathbf{Z}[x]$

$$
f(0) \equiv 1 \quad(\bmod 2)
$$

$u$ для любого $z \in \mathbf{Z}$

$$
\begin{aligned}
f^{\prime}(z) & \equiv 1 \quad(\bmod 4) \\
f^{\prime \prime}(z) & \equiv 1 \quad(\bmod 4), \\
f(z+1) & \equiv f(z)+1 \quad(\bmod 4) .
\end{aligned}
$$

1.2. По поводу последнего результата необходимо отметить, что условия предложения 3 не являются необходимыми, так как, например, для полинома

$$
f(x)=4 x^{3}+2 x^{2}+3 x+1
$$

и $m=2^{e}$ последовательность (1) имеет период, равный $m=2^{e}$, но

$$
f^{\prime}(z)=12 z^{2}+4 z+3 \equiv 3 \quad(\bmod 4)
$$

Таким образом, предложения 1, 2 и 3 дают лишь частичное решение рассматриваемой задачи. В настоящей статъе предлагается ее полное решение.

\section{2. Определения и обозначения}

2.1. Всякий полином $f(x) \in \mathbf{Z}[x]$ индуцирует корректно определенное преобразование кольца $\mathbf{Z} / m$ вычетов по модулю $m$

$$
[a]_{m} \rightarrow[f(a)]_{m}
$$

здесь $[a]_{m}$ - класс вычетов по модулю $m$, содержащий $a \in \mathbf{Z}$.

Преобразование (2) будем обозначать символом $\langle f(x) ; m\rangle$.

Отношение $a \equiv b(\bmod m)$ будем записывать также в виде

$$
a \equiv_{m} b .
$$

Определение 1. Полином $f(x) \in \mathbf{Z}[x]$ называется

- тождеством по модулю $m$, если $f(a) \equiv 0(\bmod m)$, для любого $a \in \mathbf{Z}$;

- биективным по модулю $m$, если преобразование $\langle f(x) ; m\rangle$ является подстановкой на $\mathbf{Z} / m$;

- транзитивным по модулю $m$, если подстановка $\langle f(x) ; m\rangle$ является полным циклом.

Если $f(x)$ и $g(x)$ - полиномы над $\mathbf{Z}$ от переменной $x$, то выражение

$$
f(x) \equiv g(x) \quad(\bmod m)
$$

означает, что $f(a) \equiv g(a)(\bmod m)$ для любого $a \in \mathbf{Z}$, то есть полином $f(x)-g(x)$ есть тождество по модулю $m$. 
Преобразование $\psi: \mathbf{Z} / m \rightarrow \mathbf{Z} / m$ будем называть полиномиальным по модулю $m$, если $\psi=\langle f(x) ; m\rangle$ для подходящего $f(x) \in \mathbf{Z}[x]$.

Очевидно, что все полиномиальные по модулю $m$ подстановки образуют группу, которую будем обозначать $G(m)$.

Множество всех полных циклов из $G(m)$ обозначим $H(m)$.

Очевидно, что последовательность (1) имеет период $m$ тогда и только тогда, когда полином $f(x)$ транзитивен по модулю $m$, что, в свою очередь, равносильно включению $\langle f(x) ; m\rangle \in H(m)$.

2.2. Пусть $m=p^{i} q^{j} r^{k} \ldots$, где $p, q, r, \ldots$ - различные простые числа. Тогда

$$
\mathbf{Z} / m=\mathbf{Z} / p^{i} \oplus \mathbf{Z} / q^{j} \oplus \mathbf{Z} / r^{k} \oplus \ldots,
$$

где $\oplus-$ символ прямой суммы колец. Поэтому полином $f(x) \in \mathbf{Z}[x]$ является тождеством, биективным или транзитивным по модулю $m$ тогда и только тогда, когда $f(x)$, соответственно, есть тождество, биективен или транзитивен по каждому из модулей $p^{i}, q^{j}, r^{k}, \ldots$ Сказанное означает, что, в частности,

$$
\begin{aligned}
|G(m)| & =\left|G\left(p^{i}\right)\right|\left|G\left(q^{j}\right)\right|\left|G\left(r^{k}\right)\right| \ldots, \\
|H(m)| & =\left|H\left(p^{i}\right)\right|\left|H\left(q^{j}\right)\right|\left|H\left(r^{k}\right)\right| \ldots
\end{aligned}
$$

Таким образом, рассматриваемая задача описания транзитивных по модулю $m$ полиномов сводится к случаю примарного $m$, то есть к случаю, когда $m=p^{n}$, где $p$ - простое число.

\section{3. Предварительные результаты}

Пользуясь формулой Тейлора, несложно получить, что для любых $a \in \mathbf{Z}$ и $f(x) \in \mathbf{Z}[x]$ справедливы соотношения

$$
\begin{array}{rlrl}
f\left(x+a p^{n-1}\right) & \equiv f(x)+a p^{n-1} f^{\prime}(x) \quad\left(\bmod p^{n}\right), & n \geqslant 2, \\
f\left(x+a p^{n-1}\right) & \equiv f(x)+a p^{n-1} f^{\prime}(x) \quad\left(\bmod p^{n+1}\right), & n \geqslant 3, \\
f(x+a p) & \equiv f(x)+a p f^{\prime}(x)+a^{2} p^{2} f^{\prime \prime}(x) / 2 \quad\left(\bmod p^{3}\right) . & &
\end{array}
$$

Предложение 4 ([2]). Пусть $f(x \in \mathbf{Z}[x])$. Следующие утверждения равносильны:

- $f(x)$ биективен по модулю $p^{n}$ для любого натурального n;

- $f(x)$ биективен по модулю $p^{2}$;

- $f(x)$ биективен по модулю $p$ и $f^{\prime}(a) \neq 0(\bmod p)$ для любого $a \in \mathbf{Z}$.

Предложение 5. Пусть $t(x)-$ тождество по модулю $p^{2}$. Тогда $t^{\prime}(x)-$ тождество по модулю р.

Доказательство. Для любого $c \in \mathbf{Z}$ полином $h(x)=x+c t(x)$ биективен по модулю $p^{2}$, так как $\left\langle h(x) ; p^{2}\right\rangle=\left\langle x ; p^{2}\right\rangle$. По предложению $4 h^{\prime}(a)=1+c t^{\prime}(a) \neq 0(\bmod p)$ для любых $a, c \in \mathbf{Z}$. Следовательно, $t^{\prime}(a) \equiv 0(\bmod p)$ для любого $a \in \mathbf{Z}$, то есть $t^{\prime}(x)-$ тождество по модулю $p$. 
Для $h(x) \in \mathbf{Z}[x]$ и $k \geqslant 0$ положим $h^{(0)}(x)=x$ и $h^{(k)}(x)=h\left(h^{(k-1)}(x)\right)$. Нетрудно видеть, что

$$
\left(h^{(k)}(x)\right)^{\prime}=\prod_{i=0}^{k-1} h^{\prime}\left(h^{(i)}(x)\right)
$$

Пусть $f(x) \in \mathbf{Z}[x]$ и $a \in \mathbf{Z}$. Положим $a_{1}=f^{(i)}(a), i \geqslant 0$. Легко видеть, что полином $f(x)$ транзитивен по модулю $p^{n}$ тогда и только тогда, когда последовательность $\left(a_{i}: i \geqslant 0\right)$ содержит полную систему вычетов по модулю $p^{n}$. Отсюда очевидным образом вытекает следующее утверждение.

Предложение 6. Если полином транзитивен по модулю $p^{n}$, то он транзитивен по модулю $p^{n-1}$.

Предложение 7. Пусть полином $f(x) \in \mathbf{Z}[x]$ транзитивен по модулю $p^{2}$ u $g(x)=$ $f^{\left(p^{s}\right)}(x)$, где $s \geqslant 1$. Тогда $g^{\prime}(x) \equiv 1(\bmod p)$ и если $p \neq 2$, то

$$
\sum_{i=0}^{p-1}\left(g^{\prime}(x)\right)^{i} \equiv p \quad\left(\bmod p^{2}\right)
$$

Доказательство. Так как $f(x)$ транзитивен по модулю $p^{2}$ и $s \geqslant 1$, то

$$
g^{(p)}(x)-x=f^{\left(p^{s+1}\right)}(x)-x
$$

есть тождество по модулю $p^{2}$, откуда по предложению $5\left(g^{(p)}(x)\right)^{\prime} \equiv 1(\bmod p)$. По предложению $6 g(x) \equiv x(\bmod p)$ и согласно $(6)\left(g^{(p)}(x)\right)^{\prime} \equiv\left(g^{\prime}(x)\right)^{p}(\bmod p)$, то есть $\left(g^{\prime}(x)\right)^{p} \equiv 1(\bmod p)$ и тем самым $g^{\prime}(x) \equiv 1(\bmod p)$.

Таким образом, $g^{\prime}(x)=1+p \alpha(x)$ для подходящей функщии $\alpha(x)$, откуда получаем, что $\left(g^{\prime}(x)\right)^{i} \equiv 1+i p \alpha(x)\left(\bmod p^{2}\right)$ и

$$
\sum_{i=0}^{p-1}\left(g^{\prime}(x)\right)^{i} \equiv p+p^{2}(p-1) / 2 \alpha(x) \quad\left(\bmod p^{2}\right) .
$$

Если $p \neq 2$, то, очевидно, получаем требуемое утверждение.

\section{4. Критерии транзитивности}

Предложение 8. Полином $f(x) \in \mathbf{Z}[x]$ транзитивен по модулю $p^{n}$ тогда и только тогда, когда для подходящего а $\in \mathbf{Z}$ справедлива система соотночений

$$
\begin{aligned}
f^{\left(p^{n}\right)}(a) \equiv a & \left(\bmod p^{n}\right), \\
f^{\left(p^{n-1}\right)}(a) \neq a & \left(\bmod p^{n}\right) .
\end{aligned}
$$

Доказательство. Необходимость условия (7) очевидна. Докажем достаточность. Из первого соотношения системы (7) следует, что точка $a$ (точнее, класс вычетов по модулю $p^{n}$, содержащий $a)$ лежит на цикле преобразования $\left\langle f(x) ; p^{n}\right\rangle$, длина $r$ которого делит $p^{n}$. Из второго соотношения системы (7) следует, что $p^{n-1}$ не делит $r$. Поэтому $r=p^{n}$. 
Как показывает пример полинома $x^{p}+1$, утверждение, обратное предложению 6 , неверно. Однако справедливо следующее утверждение.

Предложение 9. Если полином транзитивен по модулю $p^{3}$, то он транзитивен по модулю $p^{n}$ для любого натурального $n$.

Доказательство. Пусть $n \geqslant 3$ и $f(x)$ тразитивен по модулю $p^{n}$. Очевидно, что достаточно доказать транзитивность $f(x)$ по модулю $p^{n+1}$.

Положим $g(x)=f^{\left(p^{n-1}\right)}(x)$. По предложению 6 получаем, что $g(x)=x+t(x)$, где $t(x)$ - тождество по модулю $p^{n-1}$, не являющееся тождеством по модулю $p^{n}$, то есть $t(a)=p^{n-1} b$ для подходящих $a, b \in \mathbf{Z}$, причем $b \neq 0(\bmod p)$.

Так как $n \geqslant 3$, из (4) индукщией по $k$ легко находим, что

$$
g^{(k)}(a) \equiv_{\left(p^{n+1}\right)} a+p^{n-1} b \sum_{i=0}^{k-1}\left(g^{\prime}(a)\right)^{i}
$$

Согласно предложению 9 при $k=p \neq 2$

$$
g^{(p)}(a) \equiv a+p^{n} b \quad\left(\bmod p^{n+1}\right) .
$$

Пусть $p=2$. Так как $f(x)$ транзитивен по модулю 4, из формулы (4) получаем, что

$$
g^{\prime}(a) \equiv_{4}\left(f^{\prime}(0) f^{\prime}(1) f^{\prime}(2) f^{\prime}(3)\right)^{2^{n-3}} .
$$

Из (3) получаем, что для любых $d, e \in \mathbf{Z}$

$$
f^{\prime}(d+2 e) \equiv f^{\prime}(d)+2 e f^{\prime \prime}(d) \quad(\bmod 4)
$$

и так как все коэффициенты полинома $f^{\prime \prime}(x)$ четны, справедливо сравнение

$$
f^{\prime}(d+2 e) \equiv f^{\prime}(d) \quad(\bmod 4)
$$

Поэтому

$$
f^{\prime}(0) f^{\prime}(1) f^{\prime}(2) f^{\prime}(3) \equiv\left(f^{\prime}(0) f^{\prime}(1)\right)^{2} \equiv 1 \quad(\bmod 4) .
$$

Отсюда, $g^{\prime}(a) \equiv 1(\bmod 4)$ и $g^{\prime}(a+1) \equiv 2(\bmod 4)$. Таким образом, (8) верно для любого простого $p$.

Так как $b \neq 0(\bmod p)$, то

$$
g^{(p)}\left(a \neq a \quad\left(\bmod p^{n+1}\right)\right) .
$$

Так как $g^{\prime}(x \equiv 1(\bmod p))$ (см. доказательство предложения 7), используя (3) и (8), индукщией по $k$ легко находим, что

$$
g^{(k p)}(a) \equiv a+k b p^{n} \quad\left(\bmod p^{n+1}\right),
$$

откуда при $k=p$ получаем, что

$$
g^{\left(p^{2}\right)}(a) \equiv a \quad\left(\bmod p^{n+1}\right) .
$$

На основании определения полинома $g(x)$ и предложения 8 система соотношений (10) и (9) равносильна транзитивности полинома $f(x)$ по модулю $p^{n+1}$. 
Нетрудно убедиться, что полином $2 x^{3}+3 x+5$ транзитивен по модулю 4 , но не является транзитивным по модулю 8 , а полином

$$
x+1-x(x-1)(x-2)(x-3)(x-4)(x-6)(x-7)
$$

транзитивен по модулю 9, но не является транзитивным по модулю 27. Следовательно, если $p \in\{2,3\}$, то результат предложения 9 усилить нельзя. Однако справедливо следующее утверждение.

Предложение 10. Если $p \notin\{2,3\}$, то полином, транзитивный по модулю $p^{2}$, транзитивен по модулю $p^{n}$ для любого натурального п.

Доказательство. На основании предложения 9 достаточно доказать, что полином транзитивен по модулю $p^{3}$.

Положим $g(x)=f^{(p)}(x)$. По предложению $7 g^{\prime}(x) \equiv 1(\bmod p)$, а в силу предложения $6 g(x)=x+p \beta(x)$, где $\beta(a) \neq 0(\bmod p)$ для подходящего $a \in \mathbf{Z}$.

Пользуясь формулой (5), индукцией по $k$ легко доказать, что

$$
g^{(k)}(x) \equiv_{p^{3}} x+p \beta(x) \sum_{j=0}^{k-1} g^{\prime}(x)^{j}+p^{2} \beta(x)^{2}\left(g^{\prime \prime}(x) / 2\right) \sum_{j=0}^{k-1} j^{2} .
$$

Так как $p \notin\{2,3\}$, то

$$
\sum_{j=0}^{p-1} j^{2}=(p-1) p(2 p-1) / 6 \equiv 0 \quad(\bmod p),
$$

откуда, полагая в (11) $k=p$ и $x=a$, по предложению 7 получаем, что

$$
g^{(p)}(a) a+p^{2} \beta(a) \quad\left(\bmod p^{3}\right) .
$$

Так как $\beta(a) \neq 0(\bmod p)$, то

$$
g^{(p)}(a) \neq a \quad\left(\bmod p^{3}\right) .
$$

Так как $g^{\prime}(x) \equiv 1(\bmod p)$ и $\left(g^{(p)}(x)\right)^{\prime} \equiv 1(\bmod p)$ (см. предложение 7 и его доказательство), индукцией по $k$ легко находим, что

$$
g^{(k p)}(a) \equiv a+k p^{2} \beta(a) \quad\left(\bmod p^{3}\right),
$$

откуда при $k=p$ получаем, что

$$
g^{\left(p^{2}\right)}(a) \equiv\left(\bmod p^{3}\right) .
$$

На основании определения полинома $g(x)$ и предложения 8 система соотношений (13) и (12) равносильна транзитивности полинома $f(x)$ по модулю $p^{3}$.

Результат предложения 10 усилить нельзя: $f(x)=1+2 x-x^{p}$ транзитивен по модулю $p$, а при $p \neq 2$ даже биективен по модулю $p^{2}$, но так как $f^{\prime}(x) \equiv 2(\bmod p)$, для $g(x)=f^{(p)}(x)$ справедливо соотношение

$$
g^{\prime}(x)=\prod_{j=0}^{p-1} f^{\prime}\left(f^{(j)}(x)\right) \equiv 2^{p} \equiv 2 \quad(\bmod p)
$$

откуда по предложению $7 f(x)$ не является транзитивным по модулю $p^{2}$. 


\section{5. Число полноцикловых подстановок в группах $G\left(p^{n}\right)$}

Пусть $\pi_{0}(x)=1, \pi_{1}(x)=x$ и $\pi_{k}(x)=x(x-1) \ldots(x-k+1)$ для $k \geqslant 2$. В соответствии с интерполяционной формулой Ньютона любой полином $f(x) \in \mathbf{Z}[x]$ однозначно представляется в виде конечной суммы

$$
f(x)=\sum_{k \geqslant 0} c_{k} \pi_{k}(x),
$$

где все $c_{k} \in \mathbf{Z}$.

5.1. Найдем число полных циклов в группе $G\left(p^{2}\right)$.

Предложение 11 ([3]). Полином $f(x) \in \mathbf{Z}[x]$ является тождеством по модулю $m$ тогда и только тогда, когда в представлении (14) коэффичиент $c_{k}$ делится на $m /(k !, m)$ для любого $k \geqslant 0$.

Для простого $p$ и натурального $s$ положим

$$
v_{p}(s)=\max \left(\nu \geqslant 0: p^{v} \text { делит } s\right),
$$

и пусть

$$
\varkappa(p, n)=\min \left(k \geqslant 0: v_{p}(k !) \geqslant n\right) .
$$

Предложение 12 ([4]). Порядок группы $G\left(p^{n}\right)$ равен $p !$, если $n=1, p !(p-1)^{p} p^{p}$, если $n=2, u$

$$
p !(p-1)^{p} p^{p+\varkappa(p, 3)+\ldots+\varkappa(p, n)},
$$

если $n \geqslant 3$.

Как показано в [4], при $n \geqslant 2$ отображение

$$
G\left(p^{n}\right) \rightarrow G\left(p^{n-1}\right),
$$

действующее по правилу

$$
\left\langle f(x), p^{n}\right\rangle \rightarrow\left\langle f(x), p^{n-1}\right\rangle,
$$

определено корректно и является эпиморфизмом групп.

Ядро указанного эпиморфизма обозначим $K\left(p^{n}\right)$. Согласно предложению 12 порядок $K\left(p^{n}\right)$ равен

$$
\begin{array}{r}
(p-1)^{p} p^{p}, \text { если } n=2, \\
p^{\varepsilon(p, n)}, \text { если } n \geqslant 3 .
\end{array}
$$

Пусть $H\left(p^{2}\right)$ - множество всех полных циклов из $G\left(p^{2}\right)$. Если

$$
\sigma=\left\langle f(x), p^{2}\right\rangle \in H\left(p^{2}\right),
$$

то по предложению $6\langle f(x), p\rangle \in H(p)$, и поэтому

$$
H\left(p^{2}\right) \subset \bigcup_{\sigma} \sigma K\left(p^{2}\right) .
$$


где объединение смежных классов $\sigma K\left(p^{2}\right)$ берется по всем подстановкам $\sigma \in H\left(p^{2}\right)$.

Так как $G(p)$ есть симметрическая группа степени $p$, все полные циклы из $G(p)$ сопряжены в ней. Поэтому для любых подстановок $\sigma_{1}, \sigma_{2} \in H\left(p^{2}\right)$ смежные классы $\sigma_{1} K\left(p^{2}\right)$ и $\sigma_{2} K\left(p^{2}\right)$ сопряжены в $G\left(p^{2}\right)$ и, следовательно, содержат одинаковое число полных циклов.

Определим отображение

$$
\delta: G\left(p^{2}\right) \rightarrow(\mathbf{Z} / p)^{*}
$$

группы $G\left(p^{2}\right)$ в мультипликативную группу $(\mathbf{Z} / p)^{*}$ поля $\mathbf{Z} / p$ следующим образом. Пусть $\sigma=\left\langle f(x), p^{2}\right\rangle$ и $\sigma^{\prime}=\left\langle f^{\prime}(x), p^{2}\right\rangle$. Тогда

$$
\delta(\sigma)=\prod_{z} \sigma^{\prime}(z)
$$

где $z$ пробегает поле $\mathbf{Z} / p$.

Из предложений 4, 5 и правила дифференцирования сложной функции получаем, что отображение (17) определено корректно и является гомоморфизмом групп.

Если $s \neq 0(\bmod p)$, то для $f(x)=(1-s)^{p} x^{p}+s x$ подстановка $\left\langle f(x), p^{2}\right\rangle$ принадлежит $K\left(p^{2}\right)$ и $f^{\prime}(x) \equiv s(\bmod p)$. Отсюда, учитывая сравнение $s^{p} \equiv s(\bmod p)$, получаем, что гомоморфизм $\delta$ является эпиморфизмом $K\left(p^{2}\right)$ на $(\mathbf{Z} / p)^{*}$.

Таким образом, для каждого $z \in(\mathbf{Z} / p)^{*}$ в любом смежном классе $\sigma K\left(p^{2}\right)$ имеется точно $\left|K\left(p^{2}\right)\right| /(p-1)$ подстановок $\tau$, для которых $\delta(\tau)=z$.

По предложению 7 для $\sigma \in H\left(p^{2}\right)$ значения $\delta(\sigma)$ одинаковы и равны единице группы $(\mathbf{Z} / p)^{*}$. Поэтому в силу (16) справедливо неравенство

$$
\left|H\left(p^{2}\right)\right| \leqslant(p-2) !\left|K\left(p^{2}\right)\right|=\left|G\left(p^{2}\right)\right| /(p(p-1)),
$$

причем для $p=2$ неравенство (18) строгое, в чем легко убедиться на примере полинома $1-x$, который не транзитивен по модулю 4 , однако подстановка $\langle 1-x, 4\rangle$ лежит в смежном классе $\sigma K(4)$, где $\sigma=\langle x+1,4\rangle$.

Полный цикл коммутирует в любой содержащей его группе подстановок только со своими степенями, поэтому

$$
\left|H\left(p^{2}\right)\right|=r\left|G\left(p^{2}\right)\right| / p^{2},
$$

где $r$ - число классов попарно сопряженных в $G\left(p^{2}\right)$ полных циклов. Из (18) и (19) получаем, что

$$
r \leqslant p /(p-1)
$$

Если $p \geqslant 3$, то из (20) следует, что $r=1$. Если $p=2$, то неравенство (20) строгое (ибо, как отмечено выше, строгим является неравенство (18)), откуда снова $r=1$, то есть все полные циклы сопряжены в $G\left(p^{2}\right)$.

Таким образом, учитывая предложение 12 , получаем следующее утверждение.

Предложение 13. Все полные чиклы из группы $G\left(p^{2}\right)$ сопряжены в ней, и число их равно

$$
(p-2) !(p-1)^{p+1} p^{p-1} \text {. }
$$

Теперь найдем число полных циклов в группах $G\left(p^{3}\right)$ для случаев, когда $p \in\{2,3\}$. 
5.2. Рассмотрим случай $p=2$. Легко видеть, что подстановка $\left\langle 2 x^{3}+3 x+4,4\right\rangle$ лежит в $K(8)$ и является транспозицией, то есть нечетной подстановкой. Следовательно, для любого $\sigma \in G(8)$ ровно половина подстановок из смежного класса $\sigma K(8)$ нечетны. В силу (15) $|K(8)|=16$, и по предложению $13|H(4)|=2$, поэтому $|H(8)| \leqslant 16$. По предложению $12|G(8)|=2^{7}$, и, так как класс попарно сопряженных в $G(8)$ полных циклов содержит $|G(8)| / 8=16$ подстановок, то $|H(8)| \geqslant 16$. Таким образом, получаем следующее утверждение.

Предложение 14. Все полные чиклы из группы $G(8)$ сопряжены в ней, и число их равно 16.

5.3. Рассмотрим случай $p=3$. Пусть $\sigma_{1}, \sigma_{2} \in H(27)$, где $\sigma_{1}=\left\langle f_{1}(x), 27\right\rangle$ и $\sigma_{2}=\left\langle f_{2}(x), 27\right\rangle$. По предложению 13 подстановки $\left\langle f_{1}(x), 9\right\rangle$ и $\left\langle f_{2}(x), 9\right\rangle$ сопряжены в $G(9)$. Поэтому смежные классы $\sigma_{1} K(27)$ и $\sigma_{2} K(27)$ сопряжены в $G(27)$ и, следовательно, содержат одинаковое число полных циклов. Таким образом, достаточно найти число полных циклов в смежном классе $\sigma K(27)$ для $\sigma=\langle x+1,27\rangle$.

Полный прообраз (при эпиморфизме $G(27) \rightarrow G(9))$ циклической подгруппы, порожденной подстановкой $\langle x+1,9\rangle$, является (на основании (15)) 3-группой. Поэтому любой элемент из $\sigma K(27)$ является 3-элементом. Значит, полином $f(x)$ является транзитивным по модулю 27 тогда и только тогда, когда $f^{(9)}(x) \neq x(\bmod 27)$ (поскольку первое из соотношений (7) заведомо выполняется).

Любой элемент из $\sigma K(27)$ индуцируется полиномом вида

$$
f(x)=x+t(x),
$$

где $t(x)$ - тождество по модулю 9, причем, по предложению 5

$$
f^{\prime}(x) \equiv 1 \quad(\bmod 3) .
$$

На основании (3), (5) и предложения 5 легко получаем, что

$$
f^{(9)}(x) \equiv 27 x+9\left(1+t^{\prime \prime}(x)+t^{\prime \prime}(x+1)+t^{\prime \prime}(x+2)\right) \text {. }
$$

По предложению 11 любое полиномиальное по модулю 27 преобразование индуцируется полиномом вида (14), степень которого не превосходит 8. Поэтому в (21) достаточно положить

$$
t(x)=\sum_{k=0}^{8} c_{k}(9 /(k !, 9)) \pi_{k}(x),
$$

откуда прямыми вычислениями несложно получить, что

$$
t^{\prime \prime}(x)+t^{\prime \prime}(x+1)+t^{\prime \prime}(x+2) \equiv c_{8} \quad(\bmod 3),
$$

то есть

$$
f^{(9)}(x) \equiv x+9\left(1+c_{8}\right) \quad(\bmod 27) .
$$

Так как в (23) все полиномы $(9 /(k !, 9)) \pi_{k}(x)$ суть тождества по модулю 9, коэффициенты $c_{k}$ достаточно выбирать из множества $\{0,1,2\}$ (что, как легко видеть, гарантирует однозначность представления преобразования $\langle t(x), 27\rangle)$.

Таким образом, полином (21) транзитивен по модулю 27 тогда и только тогда, когда в представлении (23) (где все коэффициенты $\left.c_{k} \in\{0,1,2\}\right) c_{8} \neq 2$.

Поэтому смежный класс $\sigma K(27)$, где $\sigma=\langle x+1,27\rangle$, содержит $2 \cdot 3^{8}$ полных циклов. Учитывая, что порядок централизатора полного цикла из $H(27)$ равен 27 , а также, что по предложению $13|H(9)|=2^{4} \cdot 3^{2}$, получаем следующее утверждение. 
Предложение 15. Число полночикловых подстановок в группе $G(27)$ равно $2^{5} \cdot 3^{10}$.

5.4. Положим для простого $p$

$$
\mu=\mu(p)= \begin{cases}2, & \text { если } p \notin\{2,3\} \\ 3, & \text { если } p \in\{2,3\} .\end{cases}
$$

Тогда по предложениям 9, 10 получаем, что при $n \geqslant \mu$ из того, что $\left\langle f(x), p^{n}\right\rangle \in H\left(p^{n}\right)$, следует, что $\sigma=\left\langle f(x), p^{n+1}\right\rangle \in H\left(p^{n+1}\right)$ и смежный класс $\sigma K\left(p^{n+1}\right)$ состоит только из полноцикловых подстановок. Поэтому справедливо следующее утверждение.

Предложение 16. Число полночикловых подстановок в группе $G\left(p^{n}\right)$ всех полиномиальных подстановок кольча $\mathbf{Z} / p^{n}$ равно $(p-1)$ !, если $n=1,(p-2) !(p-1)^{p+1} p^{p-1}$, если $n=2,(p-2) !(p-1)^{p+1} p^{p-1+\varepsilon(p, 3)+\ldots+\varepsilon(p, n)}$, если $n \geqslant 3$ и $p \notin\{2,3\}$, равно 16, если $p^{n}=8,2^{5} 3^{10}$, если $p^{n}=27,2^{4+\varepsilon(2,4)+\ldots+\varepsilon(2, n)}$, если $n \geqslant 4 u p=2, u 2^{5} 3^{10+\varepsilon(3,4)+\ldots+\varepsilon(3, n)}$, если $n \geqslant 4$ и $p=3$.

\section{6. Описание транзитивных полиномов}

6.1. Пусть $\sigma \in H\left(p^{2}\right)$ и $C(\sigma)$ - централизатор $\sigma$ в $G\left(p^{2}\right)$. Так как $C(\sigma)$ - циклическая подгруппа, порожденная $\sigma$, а $\sigma$ - полный цикл, ясно, что для любых $z, w \in \mathbf{Z} / p^{2}$ в $C(\sigma)$ существует единственная подстановка $\tau$ такая, что $\tau(z)=w$. Отсюда следует, что для любой подстановки $\omega \in G\left(p^{2}\right)$ в смежном классе $\omega C(\sigma)$ существует единственная подстановка $\psi$ такая, что $\psi(0)=0$ (здесь $0-$ нуль кольца $\mathbf{Z} / p^{2}$ ).

Обозначим через $G_{0}\left(p^{2}\right)$ подгруппу $\left\{\psi \in G\left(p^{2}\right): \psi(0)=0\right\}$ группы $G\left(p^{2}\right)$. По предложению 13 в совокупности

$$
\left\{\psi^{-1} \sigma \psi: \psi \in G_{0}\left(p^{2}\right)\right\}
$$

встречается каждый полный цикл из $G\left(p^{2}\right)$, причем точно по одному разу.

6.2. Пусть $A$ - полная система вычетов по модулю $p$, причем $A \subset\left\{0,1, \ldots, p^{2}-1\right\}$, $\alpha: Z / p \rightarrow A-$ сюръекция, $\beta: Z / p \rightarrow(\mathbf{Z} / p)^{*}$.

Каждый элемент $z \in \mathbf{Z} / p^{2}$ однозначно представляется в виде $z=u+p v$, где $0 \leqslant$ $u, v \leqslant p-1$. Определим преобразование $\gamma$ кольца $\mathbf{Z} / p$, положив

$$
\gamma(u+p v)=\alpha(u)+p v \beta(u) .
$$

Очевидно, что множество $A$ можно выбрать $p^{p}$ различными способами, для каждого $A$ существует $p$ ! различных сюръекций $\alpha$, существует $(p-1)^{p}$ различных отображений $\beta$, отображение $\gamma$ биективно на $\mathbf{Z} / p^{2}$.

Таким образом, существует точно

$$
p !(p-1)^{p} p^{p}
$$

различных биективных на $\mathbf{Z} / p^{2}$ отображений вида (25).

Пусть $f(x)$ - биективный по модулю $p^{2}$ полином из $\mathbf{Z}[x]$. Обозначим через $\alpha$ сужение на $\mathbf{Z} / p$, а через $\beta$ отображение $\langle f(x), p\rangle$. Тогда по формуле (3) находим, что $\gamma=\left\langle f(x), p^{2}\right\rangle$. 
Таким образом, любое полиномиальное преобразование, биективное по модулю $p^{2}$, имеет вид (25). Так как величина (26) совпадает с порядком группы $G\left(p^{2}\right)$, справедливо следующее утверждение.

Предложение 17. Любое преобразование кольча $\mathrm{Z} / p^{2}$, имеющее вид (25), полиномиально.

На основании сказанного сразу получаем следующее описание множества $H\left(p^{2}\right)$.

Предложение 18. Пусть $\sigma=\left\langle x+1, p^{2}\right\rangle u G_{0}\left(p^{2}\right)$ - группа всех подстановок $\gamma$ из $G\left(p^{2}\right)$ вида (25) с условием $\gamma(0)=0$. Тогда

$$
H\left(p^{2}\right)=\left\{\gamma^{-1} \sigma \gamma: \gamma \in G_{0}\left(p^{2}\right)\right\}
$$

Пусть $R\left(p^{2}\right)$ - множество, состоящее из $\left|H\left(p^{2}\right)\right|$ полиномов $f(x)$, индуцирующих все подстановки из $H\left(p^{2}\right)$. Множество $R\left(p^{2}\right)$ можно найти, решив относительно коэффициентов $c_{k}$ треугольную систему уравнений, которая получается из (14), при этом ясно, что достаточно брать $k \leqslant 2 p-1$.

6.3. При доказательстве предложения 15 были найдены (см. (21), (23)) полиномиальные представления множества

$$
H_{1}(27)=(\langle x+1,27\rangle K(27)) \cap H(27)
$$

всех полных циклов $\langle f(x), 27\rangle$ таких, что $\langle f(x), 9\rangle=\langle x+1,9\rangle$.

Теперь для получения множества $R(27)$, состоящего из $|H(27)|$ полиномов, индуцирующих все подстановки из $H(27)$, можно действовать следующим образом:

- по известным полиномиальным представлениям найти все подстановки из $H_{1}(27)$;

- по известным (см. (25)). подстановкам из $G_{0}(9)$ найти (см. раздел 6.2) множество $\left\{f_{i}(x): 1 \leqslant i \leqslant 144\right\}$ индуцирующих эти подстановки полиномов (заметим, что $\left.\left|G_{0}(9)\right|=|H(9)|=2^{4} 3^{2}=144\right)$;

- найти подстановки $\sigma=\left\langle f_{i}(x), 27\right\rangle$, где $1 \leqslant i \leqslant 144$;

- тогда на основании предложения 13

$$
H(27)=\sum_{j=1}^{144} \sigma_{j}^{-1} H_{1}(27) \sigma_{j}
$$

- по подстановкам из $H(27)$ найти $|H(27)|$ индуцирующих эти подстановки полиномов (см. раздел 6.2).

6.4. Легко проверить, что для $H(8)$ соответствующее множество $R(8)$ состоит из следующих 16 полиномов:

$$
\begin{array}{llll}
x+1, & 5 x+1, & 2 x^{2}+3 x+1, & 2 x^{2}+7 x+1, \\
x+3, & 5 x+3, & 2 x^{2}+3 x+3, & 2 x^{2}+7 x+3, \\
x+5, & 5 x+5, & 2 x^{2}+3 x+5, & 2 x^{2}+7 x+5 \\
x+7, & 5 x+7, & 2 x^{2}+3 x+7, & 2 x^{2}+7 x+7 .
\end{array}
$$


6.5. На основании предложений 9-11, а также результатов разделов 6.1-6.4 и 5.4 сформулируем окончательный результат.

Предложение 19. Полином $f(x) \in \mathbf{Z}[x]$ транзитивен по модулю $p^{n}$ для любого натурального п тогда и только тогда, когда $f(x)$ представляется в виде

$$
f(x)=r(x)+t(x)
$$

где $r(x) \in R\left(p^{\mu}\right)$, a $t(x)$ - тождество по модулю $p^{\mu}$ (здесь $\mu=2$ при $p \notin\{2,3\}$ u $\mu=3$ nрu $p \in\{2,3\})$.

\section{7. О транзитивных полиномах по модулю $2^{n}$}

Для $p=2$ можно получить необходимые и достаточные условия транзитивности по модулю $2^{n}$ полинома в виде системы линейных соотношений между его коэффициентами.

Предложение 20. Полином $f(x)=a x^{3}+b x^{2}+c x+d \in \mathbf{Z}[x]$ транзитивен по модулю 8 тогда и только тогда, когда выполнена следующая система соотношений:

$$
\begin{aligned}
a \equiv 0 & (\bmod 4), \\
b \equiv 0 & (\bmod 2), \\
b+c \equiv 1 & (\bmod 4), \\
d \equiv 1 & (\bmod 2) .
\end{aligned}
$$

Доказательство. На основании результатов раздела 6.4 среди полиномов (27) существует такой полином $g(x)$, что полином $t(x)=f(x)-g(x)$ является тождеством по модулю 8. Так как $\operatorname{deg} g(t) \leqslant 2$, старшие коэффициенты полиномов $t(x)$ и $f(x)$ совпадают, и поскольку $\operatorname{deg} f(x) \leqslant 3$, из предложения 11 для $m=8$ следует, что $a \equiv 0(\bmod 4)$. В силу очевидного соотношения $4 x^{3} \equiv 4 x(\bmod 8)$

$$
f(x) \equiv h(x)=b x^{2}+(a+c) x+d \quad(\bmod 8) .
$$

По предложению 2 полином $h(x)$ транзитивен по модулю 8 тогда и только тогда, когда выполнена система соотношений

$$
\begin{aligned}
b & \equiv 0 \quad(\bmod 2) \\
a+b+c & \equiv 1 \quad(\bmod 4), \\
d & \equiv 1 \quad(\bmod 2) .
\end{aligned}
$$

Так как $a \equiv 0(\bmod 4)$, из (29) получаем $(28)$.

Если верно $(28)$, то $a \equiv 0(\bmod 4)$ и тогда верно (29), откуда по предложению 2 полином $h(x)$ транзитивен по модулю 8 . Поскольку $4 x^{3} \equiv 4 x(\bmod 8)$, справедливо сравнение $h(x) \equiv f(x)(\bmod 8)$, откуда $f(x)$ транзитивен по модулю 8.

Пусть

$$
f(x)=\sum_{k \leqslant 0} a_{k} x^{k}
$$


Нетрудно видеть, что при $k \geqslant 2$

$$
\begin{aligned}
x^{2 k+1} & \equiv x^{3} \quad(\bmod 8) \\
x^{2 k} & \equiv x^{4} \quad(\bmod 8) \\
x^{4} & \equiv 6 x^{3}+5 x^{2}+6 x \quad(\bmod 8)
\end{aligned}
$$

Поэтому

$$
f(x) \equiv_{8}(A-2 B) x^{3}+\left(a_{2}-3 B\right) x^{2}+\left(a_{1}-2 B\right) x+a_{0},
$$

где $A=a_{3}+a_{5}+a_{7}+\ldots$ и $B=a_{4}+a_{6}+a_{8}+\ldots$ Отсюда после очевидных преобразований на основании предложения 20 получаем следующее утверждение.

Предложение 21. Полином

$$
\sum_{k \geqslant 0} a_{k} x^{k} \in \mathbf{Z}[x]
$$

транзитивен по модулю $2^{n}$ для всех $n \geqslant 1$ тогда и только тогда, когда выполнена система соотношений

$$
\begin{aligned}
a_{3}+a_{5}+a_{7}+\ldots & \equiv 2 a_{2} \quad(\bmod 4), \\
a_{4}+a_{6}+a_{8}+\ldots & \equiv a_{1}+a_{2}-1 \quad(\bmod 4), \\
a_{1} & \equiv 1 \quad(\bmod 2), \\
a_{0} & \equiv 1 \quad(\bmod 2) .
\end{aligned}
$$

\section{8. Заключение}

Результаты настоящей статьи изложены в соответствии с ситуацией, имевшей место на момент их получения. С тех пор прошло почти 17 лет, в течение которых был получен ряд новых результатов по полиномиальной транзитивности над кольцами, наиболее серьезные из которых, в том числе обобщающие предлагаемые здесь, принадлежат В. С. Анашину (см., например, его обзорную статью [5]), коему автор признателен за полезные замечания, сделанные при подготовке данной статьи к печати.

\section{Список литературы}

1. Knuth D. E., The art of computer programming. Seminumerical algorithms. V.2. Addison-Wesley, Reading, MA, 1981.

2. Nobauer W., Zur Theorie der Polinomtransformationen und Permutationspolynome. Math. Ann. (1964) 157, №4, 332-342.

3. Singmaster D., On polynomial functions $(\bmod m)$. J. Number Theory (1974) 6, №5, 345-352.

4. Keller G., Olson F. R., Counting polynomials functions $\left(\bmod p^{n}\right)$. Duke Math. J. (1968) 35, №4, 835-838.

5. Anashin V. S., Uniformly distributed sequences in computer algebra, or how to construct program generators of random numbers. J. Math. Sci. (1998) 89, №4, 1355-1390.

Статья поступила 27.04.2000.

Переработанный вариант поступил 14.06.2001. 